\title{
Modelagem e Simulação de Offloading para Computação Móvel em Nuvem
}

\author{
Luis Sérgio da Silva Júnior, Deborah V. Magalhães, Danielo G. Gomes \\ ${ }^{1}$ Grupo de Redes de Computadores, Engenharia de Software e Sistemas (GREat) \\ Programa de Pós-Graduação em Engenharia de Teleinformática (PPGETI) \\ Universidade Federal do Ceará (UFC) \\ Campus do Pici - Fortaleza, CE - Brasil \\ \{luissilva, deborah, dgomes\}@great.ufc.br
}

\begin{abstract}
Mobile Cloud Computing (MCC) uses cloud services to spread out the energy and computing resources of mobile devices. Despite these devices have grown in use, we observe a lack of models and simulation tools to study and analyse mobile devices resource limitation using cloud computing as solution. In this paper, we model a typical MCC architecture which focuses on an offloading perspective. Our model provides a development environment to validate offloading strategies without using a real cloud infrastructure. To validate our approach, we simulate the execution time of a task on a local (mobile device) and in a remote cloud. Results show that our models represent MCC scenarios with an maximum accuracy of 98.9\%. Moreover, we verified the workload algorithm complexity impacts on the offloading decisions.
\end{abstract}

Resumo. Computação Móvel em Nuvem (Mobile Cloud Computing, MCC) utiliza os serviços de nuvens a fim de estender os recursos energéticos e computacionais de dispositivos móveis. Apesar da sua presença em nosso cotidiano, observamos uma carência de modelos e simuladores para análise de problemas relacionados à escassez de recursos dos dispositivos móveis tendo as nuvens computacionais como solução em perspectiva. Neste artigo, propomos a modelagem e simulação de uma arquitetura típica de MCC compreendendo diferentes abordagens para execução de tarefas computacionais em servidores remotos (offloading). A modelagem foi concebida com vistas à provisão de um ambiente controlado para avaliação e ajuste de políticas de offloading sem a necessidade da implantação física de uma infraestrutura computacional de larga escala. A validação da proposta foi conduzida através de simulações, a partir de experimentos com imagens entre 0.3 e 8 megapixels, e cujos resultados indicam uma acurácia de até 98.9\%. Concluímos ainda que a ordem de complexidade algorítmica da carga é diretamente proporcional à probabilidade de offloading.

\section{Introdução}

Computação móvel em nuvem (Mobile Cloud Computing, MCC) baseia-se nas características de computação em nuvem [Mell and Grance 2011] para otimizar e expandir os recursos dos dispositivos móveis. Apesar do poder computacional crescente de tais dispositivos, sua natureza móvel e compacta limita-os sob o ponto de vista da autonomia energética [Justino and Buyya 2014]. 
Além da limitação de recursos, existem outros desafios em sistemas computacionais móveis (e.g. qualidade da comunicação móvel, mobilidade, sensoriamento de ambientes) [Dinh et al. 2013]. Estratégias como offloading ${ }^{1}$ e melhorias na largura de banda em função da mobilidade tentam mitigar essas dificuldades. O termo offloading refere-se ao uso de uma infraestrutura remota para execução de tarefas oriundas de um dispositivo com recursos limitados [Cuervo et al. 2010].

Apesar da vasta literatura sobre Computação Móvel, observamos uma carência de modelos e simuladores para estudo e análise dos problemas relacionados à escassez de hardware tendo as nuvens computacionais como solução em perspectiva [Ahmed 2014], [Dinh et al. 2013], [Qi and Gani 2012], [Satyanarayanan et al. 2009], [Li et al. 2013], [Cuervo et al. 2010], [Chun et al. 2011], [Kumar et al. 2013].

O objetivo geral deste artigo é modelar e simular um ambiente real de MCC. A simulação dos modelos propostos permite o ajuste, validação de políticas de gerenciamento de recursos e ideias de comportamentos de elementos de arqutetura sem a necessidade de uma infraestrutura computacional real em escala. Tendo em vista esse cenário, criamos modelos para entidades de uma arquitetura típica de MCC. Estes modelos englobam desde a representação de componentes físicos de um ambiente de $\mathrm{MCC}$, tais como dispositivos móveis, enlaces e nuvens computacionais, até as tomadas de decisão para realização de offloading estático e dinâmico.

Para validação da proposta, dois experimentos foram projetados e executados. $\mathrm{O}$ primeiro objetivou a simulação do modelo de offloading estático e respectiva comparação com cenário real descrito em [Costa et al. 2014]; o segundo orientou-se a tomadas de decisão de offloading dinâmico baseadas em critérios estabelecidos por [Kumar et al. 2013].

As principais contribuições deste artigo são (i) uma modelagem original de entidades de uma arquitetura de MCC, com respectiva validação em simulação, (ii) uma implementação de duas estratégias de offloading (estático e dinâmico), (iii) detecção de associações de interferência entre complexidade algorítmica e procedimentos de offloading.

\section{Trabalhos Relacionados}

Vários trabalhos sobre Computação Móvel em Nuvem têm sido propostos nos últimos 5 anos. Alguns destes trabalhos são brevemente discutidos a seguir, os quais estão organizadas em propostas de arquiteturas, frameworks e modelagem analítica para MCC.

Em uma visão geral típica de MCC, os dispositivos móveis utilizam-se de nuvens computacionais como extensões de sua capacidade de processamento e armazenamento [Qi and Gani 2012], [Dinh et al. 2013] (vide Figura 1). Nela existem componentes e operações essenciais em um contexto MCC: (i) dispositivos móveis (ii) envio e recebimento de dados (iii) mecanismo de offloading (iv) nuvem computacional.

Satyanarayanan et al. 2009 apresentaram o conceito de Cloudlet, o qual pode ser entendido como uma nuvem computacional disponível através de uma rede local sem fio. O uso de Cloudlets aplica-se a ambientes de conectividade limitada ou inexistente

\footnotetext{
${ }^{1}$ Outros termos equivalentes: Augmented execution, cyber forarging ou remote execution
} 


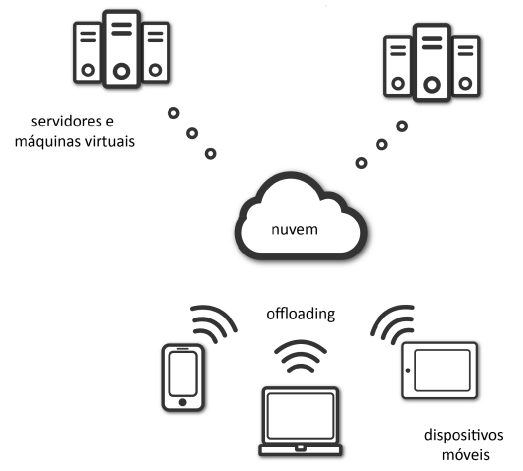

Figura 1. Visão geral MCC. Adaptada de [Qi and Gani 2012].

(e.g. ambientes fechados ou distantes de redes móveis) e no uso de aplicações sensíveis à latência.

Fernando et al. 2013 propuseram uma arquitetura que explora a utilização dos próprios dispositivos móveis disponíveis em rede para a alocação de tarefas. [Li et al. 2013] propuseram uma arquitetura com aspectos de mobilidade e cuja tomada de decisão para offloading leva em conta a mudança de localização de um dispositivo ao longo do tempo.

Alguns exemplos de frameworks foram propostos por [Cuervo et al. 2010] e [Chun et al. 2011]. Estes trabalhos utilizam ferramentas (e.g. MAUI, Clonecloud) que implementam características encontradas em MCC, tais como tomada de decisão de offloading. Essas ferramentas gerenciam recursos os quais vão desde a virtualização de uma sistema operacional em uma máquina virtual remota até a reescrita ou edição de um aplicativo móvel. Assim, para o(a) leitor(a) interessado(a) em reusar esses frameworks ou replicar os resultados apresentados nos respectivos artigos, é necessário conhecer ferramentas de manuseio de máquinas virtuais (Virtual Machines, VM) e/ou de edição de aplicativos para dispositivos móveis.

Kumar et al. 2013 propuseram uma modelagem de offloading dinâmico em que a largura de banda e a quantidade de computação das tarefas envolvidas são métricas para a tomada de decisão.

\section{Proposta}

Neste artigo propomos a modelagem e simulação de uma arquitetura típica de MCC (vide Figura 1). A modelagem proposta contempla os aspectos de offloading dos tipos estático e dinâmico [Kumar et al. 2013] [Dinh et al. 2013]. Neste sentido, propomos a criação de cinco modelos: (i) dispositivo móvel em contexto MCC (ii) tarefa passível de offloading, (iii) mecanismo de offloading estático e dinâmico, (iv) banda entre dispositivos e nuvem e, (v) infraestrutura de nuvem como um pool de servidores e VMs para execução remota de procedimentos. Esses modelos representam os componentes ilustrados na Figura 1. Sua associação com a modelagem proposta é ilustrada na Figura 2, na qual é possível distinguir quais classes representam quais modelos.

O modelo de dispositivos móveis é representado pelas classes Device e DeviceTaskExecutor (Figura 2(a)). Device contém informações sobre um dado dispositivo 
como: modelo (atributo DeviceHardware) e identificador. DeviceTaskExecutor possui uma operação chamada de executeTasksOnDevice a qual é responsável por simular a execução de uma dada tarefa em um dispositivo móvel. Atrelada a um Device, a estrutura taskList contém uma lista de tarefas.

As classes Offloader, StaticOffloader e DynamicOffloader (Figura 2(b)) possuem informações sobre a natureza de offloading a que uma tarefa é submetida. Essas classes implementam e representam os modelos de mecanismos de tomada de decisão de offloading estático e dinâmico. DynamicOffloader é uma especialização com implementação de offloading dinâmico em termos da proposta de [Kumar et al. 2013] e simplificada por [Costa et al. 2014]. Seu comportamento segue a Equação 1.

$$
\frac{W}{P_{m}}>\frac{D_{u}}{V_{u}}+\frac{D_{d}}{V_{d}}
$$

em que $W$ representa a quantidade de processamento realizado por uma dada tarefa, $P_{m}$ representa o poder computacional de um dispositivo móvel, $D_{u}$ é a quantidade de dados enviados à nuvem, $D_{d}$ é quantidade de dados recebidos, $V_{u}$ significa a taxa de upload e, de maneira análoga, $V_{d}$ é a taxa de download. Outra especialização de

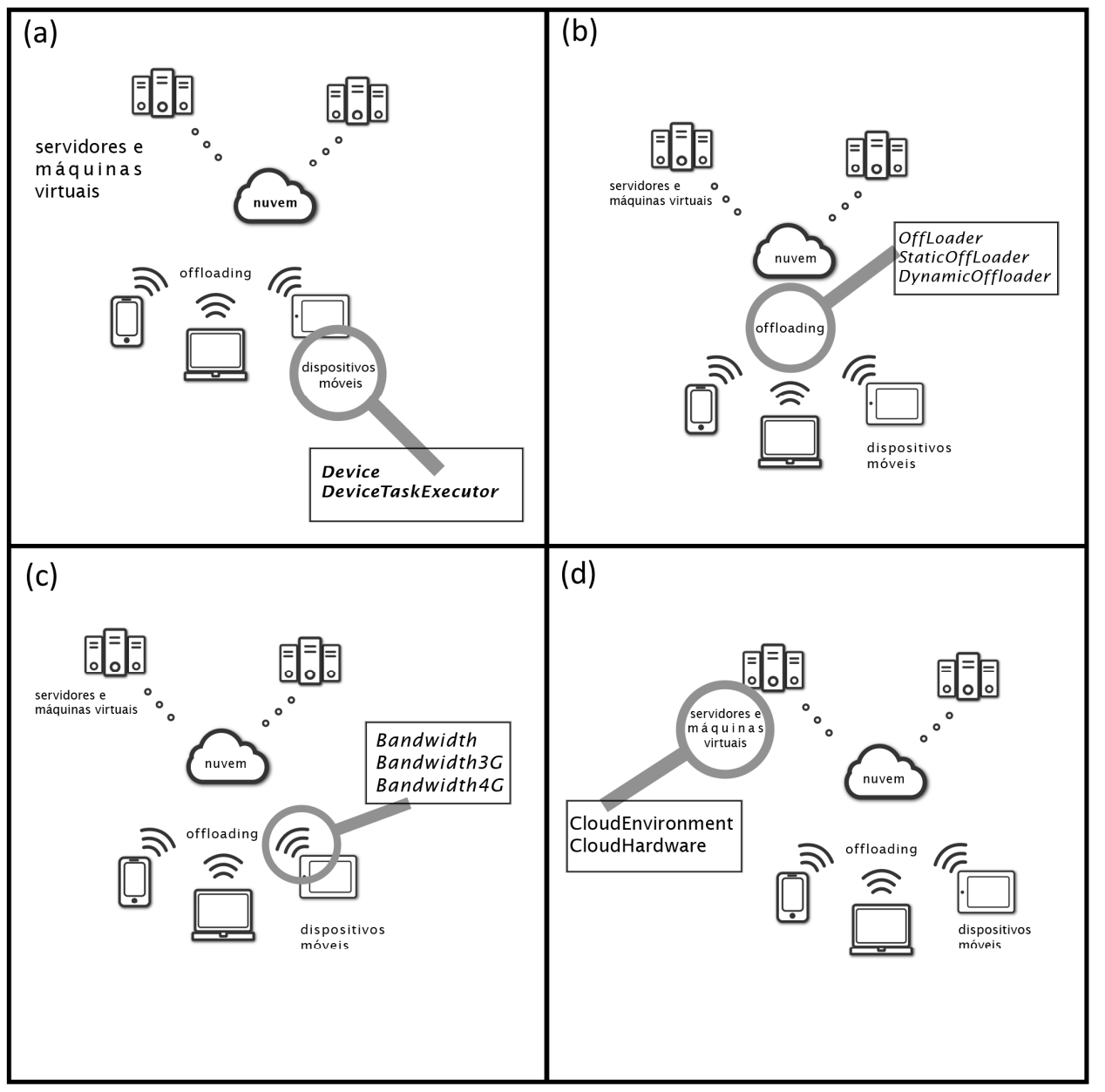

Figura 2. Associação da modelagem proposta entre as respectivas classes e (a) Dispositivos móveis, (b) Tomada de decisão de offloading, (c) Banda sem fio, (d) Infraestrutura de nuvem. 
Offloader, a classe StaticOffloader implementa uma marcação na tarefa para informar que a mesma sofrerá offloading. Ambas as especializações da classe Offloader precisam ser referenciadas por um objeto do tipo Task e isso é necessário para o cálculo do tempo total de execução do processo de offloading, independente de sua especialização, o qual é expresso pela Equação 2:

$$
T_{\text {Total }}=T_{\text {Upload }}+T_{\text {Execucao }}+T_{\text {Download }},
$$

na qual ( $T_{\text {Upload }}$ e $\left.T_{\text {Download }}\right)$ expressam o tempo de envio de informações necessárias ao offloading e recebimento de resultados oriundos da nuvem, respectivamente. $T_{\text {Execucao }}$ representa o tempo de uma tarefa executada local ou remotamente. Notar que na ausência de offloading, $T_{\text {Upload }}$ e $T_{\text {Download }}$ são nulos e somente $T_{\text {Execucao }}$ representa o tempo para execução da tarefa no dispositivo móvel.

Os modelos de banda são representados pelas entidades Bandwidth, Bandwidth3G e Bandwidth4G (Figura 2(c)) as quais são baseadas nos dados oriundos de taxas de tranmissão de dados 3G e 4G [Costa et al. 2014]. Ainda, a classe Device possui um atributo do tipo Bandwitdh (networkData) e, com sua inicialização, o dispositivo consegue calcular o tempo que uma tarefa leva ao ser realizado seu download e upload. Essa informação é utilizada durante a simulação para cálculo do tempo final de execução.

A modelagem de uma infraestrutura de nuvem é necessária para a execução de uma tarefa em uma nuvem computacional. CloudHardware e CloudEnvironment implementam esse modelo e possuem informações sobre o tempo de execução remota do procedimento e sobre a quantidade de VMs (Figura 2(d)). Neste artigo, a informação mais relevante no lado servidor é o tempo de execução remota da tarefa. Essa informação varia de acordo com o tipo de nuvem a ser considerada em simulação conforme [Costa et al. 2014]. Desta forma, nossos modelos contemplam os casos de execução em nuvens e em dispositivos móveis.

Outro aspecto a ser mencionado é a possibilidade de extensão e customização de componentes de nossa proposta como a inclusão de novos mecanismos de offloading e novas modelagens de banda e de outros dispositivos móveis.

\section{Material e Métodos}

As entidades descritas na Seção 3 foram implementadas como extensão do simulador CloudSim [Calheiros et al. 2011]. Devido à característica de representar e simular o comportamento de infraestrutura de nuvem e sua alocação de tarefas, estendemos seus componentes. Não obstante, nosso modelo está desacoplado de ferramentas e soluções externas, uma vez que nenhum componente interno do CloudSim teve seu comportamento original alterado. As entidades criadas para representar o modelo da visão geral de MCC (Figura 2) Device, Offloader, DeviceTaskExecutor, CloudEnvironment, Bandwidth e Task precisam ser instanciadas e relacionadas entre si para podermos executar a simulação e experimentos.

O primeiro passo consiste na instanciação do dispositivo móvel. Em seguida, a tarefa a ser executada na nuvem deve ser definida e instanciada. De posse desta, é necessário definir uma instância responsável pela banda que é utilizada como referência na simulação. 
Imediatamente o mecanismo de offloading (estático ou dinâmico) é definido. Depois é necessária a inclusão de informações sobre a nuvem e sua relação com a tarefa a ser executada (relação CloudEnvironment e Task). Entre elas destacamos tempo de execução da tarefa na nuvem.

Após todas as entidades serem instanciadas e devidamente relacionadas entre si a execução da simulação é realizada. Dependendo da instância de offloading inicializada, a entidade faz a simulação de um procedimento executando remota ou localmente. Finalmente, os dados gerados são passados por saídas comuns em linha de comando. Essa saída possui o tempo total de execução e os tempos intermediários descritos na Equação 2.

Para validação da proposta, dois experimentos foram projetados e executados em distintos contextos de realização de offloading. O Experimento \#1 foi projetado para validação da precisão no cálculo do tempo total de execução tratando exclusivamente de offloading estático. O Experimento \#2 foi projeto com o intuito de validar a estratégia adotada neste trabalho para tomada de decisão em offloading dinâmico. Âmbos estão descritos nas duas subseções seguintes.

\subsection{Experimento \#1: Tempo Total de Execução em Offloading Estático}

O Experimento \#1 utilizou uma simulação de offloading estático para delegar a execução da aplicação BenchImage ${ }^{2}$ à nuvem. Essa execução foi delegada a partir de dois modelos de smartphones para dois tipos de instância da Amazon EC2 configuradas com o Ubuntu Server 12.04 64-bit. O modelo de largura de banda de rede 4G foi utilizado no experimento para simular a comunicação entre o dispositivo móvel e a nuvem. Neste modelo, as taxas de upload e download são geradas aleatoriamente.

Neste experimento, simulamos uma aplicação de filtro de imagens Cartoonizer a um conjunto de imagens com tamanhos distintos. Os fatores dos experimentos e seus valores (níveis) estão nas Tabelas 1 e 2 . Durante a execução dos cenários, o tempo total de execução da aplicação (Equação 2) foi calculado para fins de comparação com os resultados alcançados em [Costa et al. 2014].

\subsection{Experimento \#2: Tomada de Decisão em Offloading Dinâmico}

O Experimento \#2 consistiu na tomada de decisão do offloading dinâmico com base nos seguintes fatores: quantidade de computação realizada e quantidade de comunicação dispositivo-nuvem [Kumar et al. 2013] para cada algoritmo (Binary Search, Bubble Sort, Matrix Multiplication). A comunicação entre o smartphone e a nuvem foi modelada considerando redes $3 \mathrm{G}$ e $4 \mathrm{G}$. Nestes modelos de largura de banda as taxas de upload e download são geradas aleatoriamente. Ao adicionarmos modelos com taxa de transmissão compatível com redes $3 \mathrm{G}$, consideramos que a variação desta taxa traz maior veracidade em cenários de decisão corriqueiros para os usuário de aplicativos, sobretudo no Brasil (Tabelas 1 e 2).

\section{Resultados e Discussões}

Os resultados do Experimento \#1 são apresentados na Figura 3. A Figura 3(a) ilustra o tempo total para execução de uma tarefa de processamento de imagem em uma instância

\footnotetext{
${ }^{2}$ https://play.google.com/store/apps/details?id=br.ufc.mdcc.benchimage
} 
Tabela 1. Critérios para o projeto dos experimentos

\begin{tabular}{l|l}
\hline Itens & Descrição \\
\hline Métricas & $\begin{array}{l}\text { Experimento 1: tempo total de execução; Experimento 2: ocorreu/não ocorreu migração } \\
\text { Parâmetros }\end{array}$ \\
$\begin{array}{l}\text { Experimento 1: filtro da imagem, tipo de offloading, tipo da rede; Experimento 2: tipo de } \\
\text { offloading, modelo do dispositivo móvel, tipo da instância, taxa de download e upload }\end{array}$ \\
\hline Fatores & $\begin{array}{l}\text { Experimento 1: tamanho da imagem, modelo do dispositivo móvel, tipo da instância; Experi- } \\
\text { mento 2: quantidade de comunicação, quantidade de computação, tipo da rede }\end{array}$ \\
\hline Técnica de análise & Simulação via CloudSim \\
\hline $\begin{array}{l}\text { Carga de Tra- } \\
\text { balho }\end{array}$ & $\begin{array}{l}\text { Experimento 1: BenchImage; Experimento 2: Binary Search, Bubble Sort e Matrix Multiplica- } \\
\text { tion }\end{array}$ \\
\hline
\end{tabular}

Tabela 2. Parâmetros de carga

\begin{tabular}{l|l}
\hline Parâmetros & Configuração \\
\hline Tamanho da imagem & $0.3 \mathrm{MP}, 1 \mathrm{MP}, 2 \mathrm{MP}, 4 \mathrm{MP}$ e 8MP \\
\hline Modelo do smartphone & LG Optimus G E977 e Samsung GT-i8190 Galaxy SIII Mini \\
\hline Tipo da instância & Amazon EC2 Medium e EC2 Micro \\
\hline Tipo de Rede & Experimento 1: 4G; Experimento 2: 3G e 4G \\
\hline Tipo de offloading & Experimento 1: estático; Experimento 2: dinâmico \\
\hline Experimento 2: Dados enviados & {$[200,400,600,800,1000]$ Matrix Multiplication } \\
(em KBytes) & {$[2000,4000,6000,8000]$ Binary Search e Bubble Sort } \\
\hline
\end{tabular}

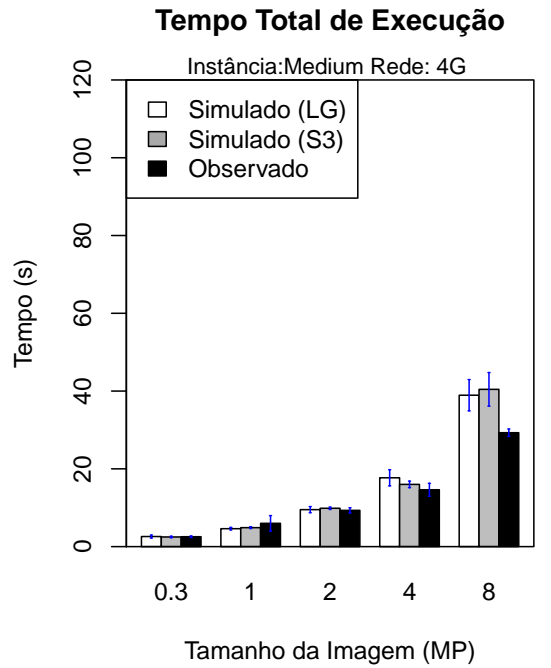

(a)

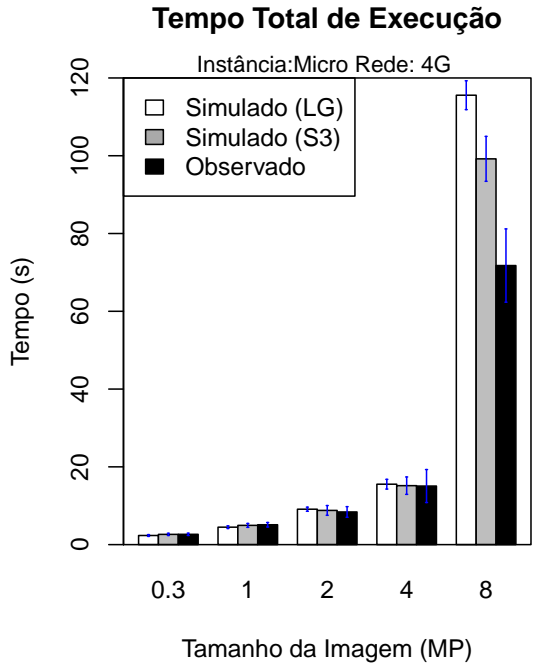

(b)

Figura 3. Experimento \#1: Tempos totais de execução (ambiente real vs. simulado), banda 4G, com diferentes tamanhos de imagem e smartphone, (a) instância Medium; (b) instância Micro.

do tipo Medium da Amazon no ambiente real e simulado. O intervalo de confiança utilizados nas simulações foi de $95 \%$.

Os resultados do experimento possuem uma acurácia de até $98.9 \%$ em comparação com valores reais obtidos em [Costa et al. 2014]. No entanto, observamos que para a imagem de 8 Megapixels, existe uma diferença de até $84 \%$ no tempo total observado e simulado. O mesmo comportamento é encontrado para a instância Micro (Figura 3(b)). Tal discrepância é explicada através da Equação 1, onde o tempo total de execução é calculado com base no tempo de download e upload das imagens. Considerando que, imagens maiores podem sofrer uma distorção maior no tempo de download e upload em relação 
a imagens menores, a perda de precisão no cálculo que pode ocorrer será maior. Outro aspecto a ser considerado é a compressão de diferentes formatos de imagem. Taxas de transmissão de dados podem sofrer influência considerando esse aspecto e, portanto, produzindo resultados ligeiramente diferentes do observado em frameworks de MCC reais.

Outro aspecto observado foi que o tempo total de execução para imagens de 8 Megapixels é bastante diferente entre os dois casos. Isso ocorre devido ao fato da capacidade de processamento da instância Medium ser maior que a da instância Micro . Essa diferença traduz-se em uma simulação que considera maior tempo de processamento para realização da tarefa em imagens maiores.

O Experimento \#2 apresenta a tomada de decisão do offloading para três algoritmos clássicos (Figuras 4 e 5), de implementações triviais e com ordens de complexidade distintas. O algoritmo Binary Search $(\theta(\log \mathrm{n}))$ pode ser utilizado em programas de buscas de dados (e.g. aplicativo de armazenamento de contatos). O algoritmo Bubble Sort $\left(\theta\left(n^{2}\right)\right)$ pode ser utilizado em programas que realizam ordenação de dados, como gerenciadores de arquivos em dispositivos móveis. Já o algoritmo Matrix Multiplication $\left(\theta\left(n^{3}\right)\right)$ pode ser utilizado em softwares de processamento de imagens e sinais de uma maneira geral.

Na Figura 4(a) observamos que, dada a mesma quantidade de comunicação, o algoritmo Bubble Sort consome um pouco mais de instruções na sua execução do que o Binary Search. Também notamos que é necessária uma quantidade de dados menor para promover a execução do algoritmo Bubble na nuvem. Portanto, observamos que a complexidade dos algoritmos impacta na tomada de decisão de offloading entre esses dois procedimentos. Tal observação é confirmada pelas Figuras 5(a) e 5(b), nas quais é apresentada a decisão de offloading do algoritmo Matrix Multiplication para as bandas 3G e 4G. Tais figuras mostram que a tomada de decisão em favor do offloading não ocorreu em apenas um casos (Matrix (local), quadrado branco, Figura 5(a)), mesmo com uma variação considerável da carga.

Apesar da escala diferir entre as Figuras 4 e 5, o modelo de tomada de decisão mostrou que o algoritmo Matrix Multiplication é bem mais suscetível a sofrer migração para execução em nuvem. Realizando uma comparação entre esses resultados e aqueles apresentados pelos algoritmos Binary Search e Bubble Sort, observamos que para uma quantidade de processamento maior, o algoritmo de offloading opta pela execução remota com pouca influência da banda da rede $3 \mathrm{G}$ ou $4 \mathrm{G}$.

Baseados nesses resultados, inferimos que algoritmos de maior grau complexidade (e.g. Matrix Multiplication) são mais suscetíveis ao offloading quando comparados a algoritmos de menor complexidade (e.g. Binary Search). Podemos ainda afirmar que o modelo produziu resultados esperados com a descrição e variação de fatores encontrados na Equação 1.

\section{Conclusões}

Este artigo apresenta uma proposta de modelagem e simulação de uma arquitetura típica de $\mathrm{MCC}^{3}$. Foram simulados dois mecanismos de decisão de offloading e, a partir dos resultados obtidos, mostrou-se que a modelagem proposta é capaz de representar cenários

\footnotetext{
${ }^{3}$ Disponível em: https://github.com/UFC-GREat-PPGETI/MCCSimulator
} 


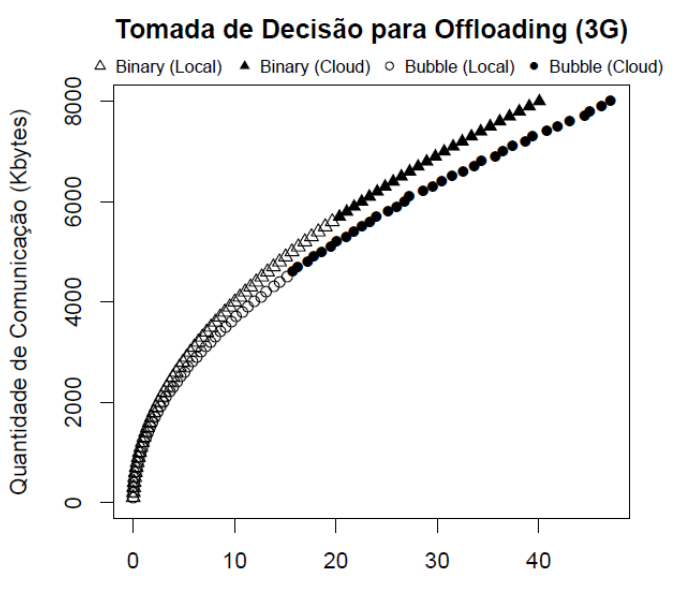

Quantidade de computação (milhões de instruções)

(a)

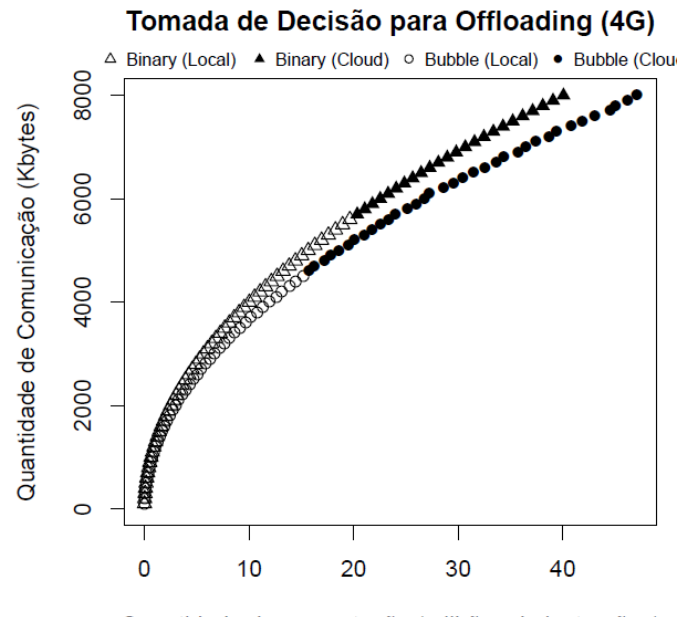

(b)

Figura 4. Experimento \#2: Decisão de offloading levando-se em conta os algoritmos Binary Search e Bubble Sort, (a) banda 3G; (b) banda 4G.

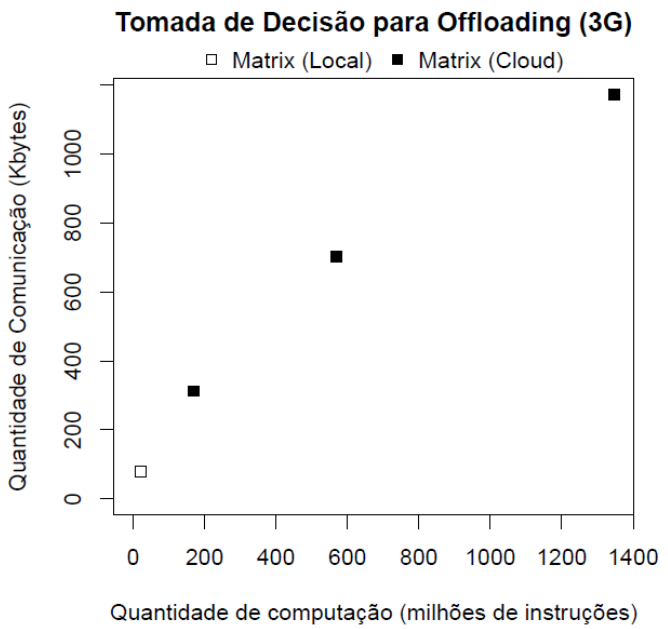

(a)

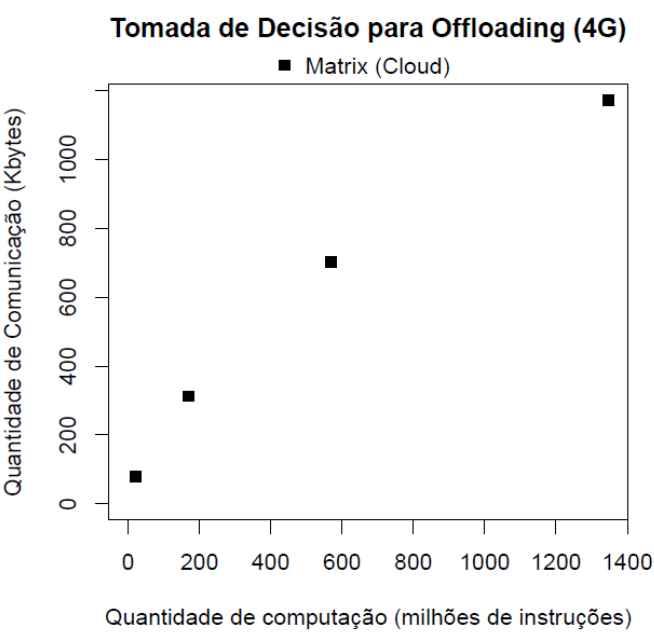

(b)

Figura 5. Experimento \#2: Decisão de offloading levando-se em conta o algoritmo Matrix Multiplication, (a) banda 3G; (b) banda 4G.

reais de offloading estático com uma acurácia de até 98.9\%. Com relação ao offloading dinâmico, foram realizadas tomadas de decisão baseadas no custo computacional e na quantidade de dados a serem transmitidos, a partir das quais concluímos que a ordem de complexidade algorítmica da carga é diretamente proporcional à probabilidade de offloading. Portanto, diante desta associação interferente, podemos concluir que a ordem de complexidade pode servir de critério de decisão em algoritmos de offloading.

Como sequência deste trabalho, podemos sugerir a inclusão de Cloudlets e de novos mecanismos de tomadas de decisão de offloading baseados no consumo energético dos dispositivos móveis. 


\section{Referências}

Ahmed, A. e Sabyasachi, A. (2014). Cloud computing simulators: A detailed survey and future direction. In Advance Computing Conference (IACC), 2014 IEEE International, pages $866-872$.

Calheiros, R. N., Ranjan, R., Beloglazov, A., De Rose, C. A. F., and Buyya, R. (2011). Cloudsim: A toolkit for modeling and simulation of cloud computing environments and evaluation of resource provisioning algorithms. Softw. Pract. Exper., 41(1):23-50.

Chun, B.-G., Ihm, S., Maniatis, P., Naik, M., and Patti, A. (2011). Clonecloud: Elastic execution between mobile device and cloud. In Proceedings of the Sixth Conference on Computer Systems, EuroSys '11, pages 301-314, New York, NY, USA. ACM.

Costa, P. B., Rego, P. A. L., Coutinho, E. F., Trinta, F. A. M., and de Souza, J. N. (2014). Uma análise do impacto da qualidade da internet móvel na utilização de cloudlets. In Simpósio Brasileiro de Redes de Computadores e Sistemas Distribuídos (SBRC 2014), pages 223-236, Florianópolis. SBC.

Cuervo, E., Balasubramanian, A., Cho, D.-k., Wolman, A., Saroiu, S., Chandra, R., and Bahl, P. (2010). Maui: Making smartphones last longer with code offload. In Proceedings of the 8th International Conference on Mobile Systems, Applications, and Services, MobiSys '10, pages 49-62, New York, NY, USA. ACM.

Dinh, H. T., Lee, C., Niyato, D., and Wang, P. (2013). A survey of mobile cloud computing: architecture, applications, and approaches. Wireless Communications and Mobile Computing.

Fernando, N., Loke, S. W., and Rahayu, W. (2013). Mobile cloud computing: A survey. Future Gener. Comput. Syst., 29(1):84-106.

Justino, T. and Buyya, R. (2014). Outsourcing resource-intensive tasks from mobile apps to clouds: Android and aneka integration. In Cloud Computing in Emerging Markets (CCEM), 2014 IEEE International Conference on, pages 1-8.

Kumar, K., Liu, J., Lu, Y.-H., and Bhargava, B. (2013). A survey of computation offloading for mobile systems. Mob. Netw. Appl., 18(1):129-140.

Li, J., Bu, K., Liu, X., and Xiao, B. (2013). Enda: Embracing network inconsistency for dynamic application offloading in mobile cloud computing. In Proceedings of the Second ACM SIGCOMM Workshop on Mobile Cloud Computing, MCC '13, pages 39-44, New York, NY, USA. ACM.

Mell, P. and Grance, T. (2011). The nist definition of cloud computing. Technical Report 800-145, National Institute of Standards and Technology (NIST), Gaithersburg, MD.

Qi and Gani, A. (2012). Research on mobile cloud computing: Review, trend and perspectives. In Digital Information and Communication Technology and it's Applications (DICTAP), 2012 Second International Conference on, pages 195-202.

Satyanarayanan, M., Bahl, P., Caceres, R., and Davies, N. (2009). The case for vm-based cloudlets in mobile computing. IEEE Pervasive Computing, 8(4):14-23. 